\title{
Best simultaneous diophantine approximations of Pisot numbers and Rauzy fractals
}

by

\author{
P. Hubert (Marseille) and A. Messaoudi (São José de Rio Preto)
}

1. Introduction. Let $v$ be an element of $\mathbb{R}^{2}, \mathcal{N}$ be a norm on $\mathbb{R}^{2}$ and $q$ an integer. We define

$$
\mathcal{N}_{0}(q v)=\min _{(p, r) \in \mathbb{Z}^{2}}\{\mathcal{N}(q v-(p, r))\} .
$$

Let $\left(q_{n}\right)_{n \geq 0}$ be a nondecreasing sequence of integers. We say that $\left(q_{n}\right)_{n \geq 0}$ is the sequence of best approximations of $v$ for the norm $\mathcal{N}$ if for all $n \in \mathbb{Z}^{+}$ and for all $0<q<q_{n}, \mathcal{N}_{0}\left(q_{n} v\right)<\mathcal{N}_{0}(q v)$.

This sequence heavily depends on the norm $\mathcal{N}$ and there is no efficient algorithm which provides the sequence of best approximations for all elements of $\mathbb{R}^{2}$. To be more precise, in the general case the sequence of best approximations is not given by any multidimensional continued fractions algorithm. It is hopeless to obtain the sequence of best approximations by such an algorithm for all real numbers (see [La82]). Nevertheless one can investigate this question for specific examples.

The situation is better understood if the coordinates of $v$ belong to a Pisot cubic field. There are fundamental tools which are available in this setting: numeration systems and fractal geometry.

The first result in this direction was obtained in [CHM01]. Let $\beta$ be the dominant root of the polynomial $X^{3}-X^{2}-X-1$. There exists a norm (called the Rauzy norm) such that the Tribonacci sequence given by

$$
\begin{gathered}
F_{0}=1, \quad F_{1}=1, \quad F_{2}=2, \\
F_{n+3}=F_{n+2}+F_{n+1}+F_{n} \quad \forall n \geq 0
\end{gathered}
$$

is the sequence of best approximations for the vector $\left(1 / \beta, 1 / \beta^{2}\right)$.

Chevallier (see [Ch99]) obtained generalizations of the previous result applying a very different method. His method works for a certain class of Pisot numbers of degree 3 with complex conjugates.

2000 Mathematics Subject Classification: 28A80, 11J70.

Research of A. Messaoudi supported by Brasilian CNPq grant, 302298/2003-7. 
In the present paper, we also generalize results of [CHM01] to a class of Pisot units of degree 3 with complex conjugates.

To each real number $\beta>1$ is naturally associated a numeration system, the $\beta$-expansion, which has been extensively studied (see [Pa60]). This $\beta$ expansion induces a $\beta$-numeration system (a sequence of integer numbers). Any integer has a unique expansion in this numeration system.

The Rauzy fractal is defined by means of a $\beta$-expansion (see [Ra82], [Ak99]) and the understanding of the geometry of this fractal is closely related to the arithmetic properties of Pisot numbers.

A Pisot number is said to have Property (F) if the $\beta$-expansion of every nonnegative element of $\mathbb{Z}[\beta]$ is finite. Pisot units of degree 3 satisfying Property (F) are classified by Akiyama (see $[\mathrm{Ak} 00]$ ). These numbers are exactly the dominant roots of the polynomials with integer coefficients

$$
x^{3}-a x^{2}-b x-1, \quad a \geq 0,-1 \leq b \leq a+1,
$$

where for $b=-1$ we add the restriction $a \geq 2$.

We prove the following result of best simultaneous diophantine approximation using Rauzy fractals:

THEOREM 1. Let $\beta$ be a non-totally real cubic Pisot unit satisfying the equation $X^{3}=a X^{2}+b X+1$; let $\left(R_{n}\right)_{n \geq 0}$ be the sequence of integers defined by

$$
\begin{aligned}
R_{0} & =1, \quad R_{1}=a, \quad R_{2}=a^{2}+b, \\
R_{n+3} & =a R_{n+2}+b R_{n+1}+R_{n} \quad \forall n \geq 0 .
\end{aligned}
$$

If $\beta$ has Property $(\mathrm{F})$, then there exists a norm $\mathcal{N}$ on $\mathbb{R}^{2}$ (called a Rauzy norm) and an integer $n_{0}$ such that $\left(R_{n}\right)_{n \geq n_{0}}$ is the sequence of best approximations of the vector $\left(1 / \beta, 1 / \beta^{2}\right)$ for the norm $\mathcal{N}$.

This result is an extension of a result of Chevallier [Ch99]. Our assumption is much weaker and has an arithmetic meaning.

We also prove a negative result when the conjugates of $\beta$ are real numbers. The geometric situation is more complicated: the Rauzy fractal is self-affine but not self similar. Theorem 2 (see Section 2) states that the behaviors of the sequences of best approximations are also very different: there is no simple generalization of Theorem 1.

\section{Background}

\subsection{Numeration systems}

$\beta$-expansion. Let $\beta>1$ be a real number. A $\beta$-representation of a nonnegative real number $x$ is an infinite sequence $\left(x_{i}\right)_{i \leq k}, x_{i} \in \mathbb{Z}^{+}$, such that

$$
x=x_{k} \beta^{k}+x_{k-1} \beta^{k-1}+\cdots+x_{1} \beta+x_{0}+x_{-1} \beta^{-1}+x_{-2} \beta^{-2}+\cdots
$$


where $k$ is a nonnegative integer. It is denoted by

$$
x=x_{k} x_{k-1} \ldots x_{1} x_{0} \cdot x_{-1} x_{-2} \ldots
$$

A particular $\beta$-representation, called the $\beta$-expansion, can be computed by the "greedy algorithm" (see [Pa60] and [F92]). Denote by $\lfloor y\rfloor$ and $\{y\}$ respectively the integer and fractional parts of a number $y$. For every real $x \geq 0$ there exists $k \in \mathbb{Z}$ such that $\beta^{k} \leq x<\beta^{k+1}$. Let $x_{k}=\left\lfloor x / \beta^{k}\right\rfloor$ and $r_{k}=\left\{x / \beta^{k}\right\}$. Then for $i<k$, put $x_{i}=\left\lfloor\beta r_{i+1}\right\rfloor$ and $r_{i}=\left\{\beta r_{i+1}\right\}$. We get

$$
x=x_{k} \beta^{k}+x_{k-1} \beta^{k-1}+\cdots .
$$

If $k<0(x<1)$, we put $x_{0}=x_{-1}=\cdots=x_{k+1}=0$. If an expansion ends with infinitely many zeros, it is said to be finite, and the final zeros are omitted.

The digits $x_{i}$ belong to the set $A=\{0, \ldots, \beta-1\}$ if $\beta$ is an integer, or to $A=\{0, \ldots,\lfloor\beta\rfloor\}$ if $\beta$ is not an integer. The $\beta$-expansion of every positive real $x$ is the lexicographically greatest among all $\beta$-representations of $x$. We will sometimes omit the splitting point between the integer part and the fractional part of the $\beta$-expansion; then the infinite sequence is just an element of $A^{\mathbb{N}}$.

For numbers $0 \leq x<1$, the expansion defined above coincides with the $\beta$-expansion of Rényi [Re57], which can be defined by means of the $\beta$ transformation of the unit interval

$$
T_{\beta}(x)=\{\beta x\}, \quad x \in[0,1] .
$$

For $x \in\left[0,1\left[\right.\right.$, we have $x_{k}=\left\lfloor\beta T_{\beta}^{k-1}(x)\right\rfloor$. However, for $x=1$ the two algorithms differ. The greedy algorithm expansion of 1 is just $1=1.0000 \ldots$, while the Rényi expansion of 1 is

$$
d(1, \beta)=. t_{-1} t_{-2} \ldots, \quad \text { where } \quad t_{-k}=\left\lfloor\beta T_{\beta}^{k-1}(1)\right\rfloor, \quad \forall k \geq 1 .
$$

Pisot numbers and Property $(\mathrm{F})$. We denote by Fin $(\beta)$ the set of numbers which have finite greedy $\beta$-expansion. For $N \in \mathbb{Z}$, we denote by $\operatorname{Fin}_{N}(\beta)$ the set of numbers $x$ such that in their $\beta$-expansion, $x_{k}=0$ for all $k<N$. We will sometimes denote a $\beta$-expansion $x_{n} \ldots x_{k}, n \geq k$, by $\left(x_{i}\right)_{n \geq i \geq k}$. We put $E_{\beta}=\left\{\left(x_{i}\right)_{i \geq k} \mid k \in \mathbb{Z}\right.$ and $\left(x_{i}\right)_{n \geq i \geq k}$ is a finite $\beta$-expansion for all $\left.n \geq k\right\}$.

We say that $\beta$ has Property (F) if

$$
\mathbb{Z}[\beta] \cap[0, \infty] \subset \operatorname{Fin}(\beta) .
$$

In what follows, we will assume that $\beta$ is a Pisot number. This means that $\beta$ is a real algebraic integer larger than 1 with its Galois conjugates of modulus strictly less than 1 .

It is known (see [Ak00]) that the class of Pisot cubic units which have Property $(\mathrm{F})$ is equal to the class of real numbers that are exactly the 
dominant roots of the following polynomials with integer coefficients:

$$
x^{3}-a x^{2}-b x-1, \quad a \geq 0,-1 \leq b \leq a+1 .
$$

(If $b=-1$ we add the restriction $a \geq 2$.)

The expansion of 1 with respect to these numbers is given by the following proposition given by Akiyama (see [Ak00]).

Proposition 1. Let $\beta$ be a Pisot cubic unit number which has Property (F) and with minimal polynomial $x^{3}-a x^{2}-b x-1$. Then

$$
d(1, \beta)= \begin{cases}. a b 1 & \text { if } 0 \leq b \leq a, \\ .(a-1)(a-1) 01 & \text { if } b=-1 \text { and } a \geq 2, \\ .(a+1) 00 a 1 & \text { if } b=a+1 .\end{cases}
$$

Lemma 1. Let $x_{n} \ldots x_{0}$ and $y_{m} \ldots y_{0}$ be two $\beta$-expansions. Then

$$
\sum_{i=0}^{n} x_{i} \beta^{i}<\sum_{i=0}^{m} y_{i} \beta^{i} \Leftrightarrow x_{n} \ldots x_{0}<_{\operatorname{lex}} y_{m} \ldots y_{0}
$$

where $<_{\operatorname{lex}}$ is the lexicographical order.

Proof. The proof is classical (see [Pa60]).

In the following, we assume that $\beta$ is a Pisot cubic unit which has Property $(\mathrm{F})$ and denote by $\alpha, \gamma$ its Galois conjugates. Let $P(x)=x^{3}-a x^{2}-b x-1$ be the minimal polynomial of $\beta$.

Numeration systems on integers. We describe the numeration systems on integers induced by $\beta$-expansions.

Assume that $d(1, \beta)=. a_{-1} \ldots a_{-t}$ where $a_{-t} \neq 0$. Let $\left(R_{n}\right)_{n \geq 0}$ be the integer sequence defined by

$$
\begin{aligned}
R_{0} & =1, \quad R_{1}=a, \quad R_{2}=a^{2}+b, \\
R_{n+3} & =a R_{n+2}+b R_{n+1}+R_{n} \quad \forall n \geq 0 .
\end{aligned}
$$

Since the polynomial $Q(x)=x^{t}-a_{-1} x^{t-1}-\cdots-a_{-t}$ is a multiple of the polynomial $x^{3}-a x^{2}-b x-1$, the sequence $\left(R_{n}\right)_{n \geq 0}$ satisfies

$$
R_{n+t}=a_{-1} R_{n+t-1}+a_{-2} R_{n+t-2}+\cdots+a_{-t} R_{n} \quad \forall n \geq 0 .
$$

Proposition 2. Every nonnegative integer $n$ has a unique $R$-ary digital expansion $\left(\varepsilon_{j}\right)_{k(n) \geq j \geq 0}$ such that $n=\sum_{j=0}^{k(n)} \varepsilon_{j} R_{j}$ with $\varepsilon_{j} \geq 0$. In particular:

1. If $a \geq b \geq 0$ and $a \neq 0$ then $\varepsilon_{i} \varepsilon_{i-1} \varepsilon_{i-2}<_{\operatorname{lex}}$ ab1 for all $i \geq 2$, $\varepsilon_{1} \varepsilon_{0}<$ lex $a b, \varepsilon_{0}<a$.

2. If $b=-1$ and $a \geq 2$ then $\varepsilon_{i} \varepsilon_{i-1} \varepsilon_{i-2} \varepsilon_{i-3}<_{\operatorname{lex}}(a-1)(a-1) 01$ for all $i \geq 3, \varepsilon_{2} \varepsilon_{1} \varepsilon_{0}<_{\operatorname{lex}}(a-1)(a-1) 0, \varepsilon_{1} \varepsilon_{0}<_{\operatorname{lex}}(a-1)(a-1), \varepsilon_{0}<a$.

3. If $b=a+1$ and $a \geq 1$ then $\varepsilon_{i} \varepsilon_{i-1} \varepsilon_{i-2} \varepsilon_{i-3} \varepsilon_{i-4}<\operatorname{lex}(a+1) 00 a 1$ for all $i \geq 4, \varepsilon_{3} \varepsilon_{2} \varepsilon_{1} \varepsilon_{0}<_{\operatorname{lex}}(a+1) 00 a, \varepsilon_{2} \varepsilon_{1} \varepsilon_{0}<_{\operatorname{lex}}(a+1) 00, \varepsilon_{1} \varepsilon_{0}<_{\operatorname{lex}}$ $(a+1) 1, \varepsilon_{0}<a$. 
4. If $a=0$ and $b=1$ then $\varepsilon_{i} \varepsilon_{i-1} \varepsilon_{i-2} \varepsilon_{i-3} \varepsilon_{i-4}<_{\text {lex }} 10001$ for all $i \geq 4$ and $\varepsilon_{0}=\varepsilon_{1}=\varepsilon_{2}=\varepsilon_{3}=\varepsilon_{5}=0$.

Proof. The digits $\left(\varepsilon_{j}\right)_{k(n) \geq j \geq 0}$ are obtained by using the greedy algorithm for integers.

1. If $a \geq b \geq 0$ and $a \neq 0$, then $\left(R_{n}\right)_{n \geq 0}$ is a nonincreasing sequence of positive integers. Hence by the definition of the greedy algorithm it is easy to see $([\mathrm{Pa} 60])$ that $\sum_{i=0}^{j} \varepsilon_{i} R_{i}<R_{j+1}$ for $0 \leq j \leq k(n)$. We deduce that

$$
\varepsilon_{j} \varepsilon_{j-1} \ldots \varepsilon_{j-t+1}<_{\mathrm{lex}} a_{-1} a_{-2} \ldots a_{-t} \quad \forall j \geq t-1 .
$$

By (1) and Proposition 1, we have $\varepsilon_{i} \varepsilon_{i-1} \varepsilon_{i-2}<_{\operatorname{lex}} a b 1$. Since $n-\sum_{i=1}^{k(n)} \varepsilon_{i} R_{i}=$ $\varepsilon_{0} R_{0}=\varepsilon_{0}<R_{1}=a$ and $n-\sum_{i=2}^{k(n)} \varepsilon_{i} R_{i}=\varepsilon_{1} R_{1}+\varepsilon_{0} R_{0}<R_{2}=a^{2}+b=$ $a R_{1}+b R_{0}$, we have $\varepsilon_{0}<a$ and $\varepsilon_{1} \varepsilon_{0}<$ lex $a b$.

2. If $b=-1$ and $a \geq 2$, then by using the same argument as in case 1 , we find that $\varepsilon_{i} \varepsilon_{i-1} \varepsilon_{i-2} \varepsilon_{i-3}<_{\operatorname{lex}}(a-1)(a-1) 01$ for all $i \geq 3$. The other conditions follow from $\varepsilon_{0} R_{0}=\varepsilon_{0}<R_{1}=a, \varepsilon_{1} R_{1}+\varepsilon_{0} R_{0}<R_{2}=$ $(a-1) R_{1}+(a-1) R_{0}$ and $\varepsilon_{2} R_{2}+\varepsilon_{1} R_{1}+\varepsilon_{0} R_{0}<R_{3}=(a-1) R_{2}+(a-1) R_{1}$.

3. If $b=a+1$ and $a \geq 1$ then by the same argument as in case 1 , we deduce that $\varepsilon_{i} \varepsilon_{i-1} \varepsilon_{i-2} \varepsilon_{i-3} \varepsilon_{i-4}<_{\operatorname{lex}}(a+1) 00 a 1$ for all $i \geq 4$. The other conditions follow from $R_{1}=a R_{0}, R_{2}=(a+1) R_{1}+R_{0}, R_{3}=(a+1) R_{2}$ and $R_{4}=(a+1) R_{3}+a R_{0}$.

4. If $a=0$ and $b=1$ then relation (1) and Proposition 1 imply that $\varepsilon_{i} \varepsilon_{i-1} \varepsilon_{i-2} \varepsilon_{i-3} \varepsilon_{i-4}<_{\text {lex }} 10001$ for all $i \geq 4$. On the other hand, $R_{0}=R_{2}=$ $R_{3}=R_{4}=1, R_{1}=0, R_{5}=R_{6}=2$. Hence $\varepsilon_{0}=\varepsilon_{1}=\varepsilon_{2}=\varepsilon_{3}=\varepsilon_{5}=0$.

LEMMA 2. For all $n \in \mathbb{Z}^{+}$we have

$$
\beta^{n}=R_{n}+\left(b R_{n-1}+R_{n-2}\right) / \beta+R_{n-1} / \beta^{2} .
$$

Proof. The proof, left to the reader, is by induction on $n$.

Let $\left(\varepsilon_{j}\right)_{k(n) \geq j \geq 0}$ be an $R$-ary digital expansion of an integer $n>0$. Let $Z=\left\{j \mid \varepsilon_{j} \neq 0\right\}, m=\min (Z)$ and $M=\max (Z)$. The number $M-m+1$ is called the length of the $R$-ary digital expansion $\left(\varepsilon_{j}\right)_{k(n) \geq j \geq 0}$.

Now, we are able to state Theorem 2.

Theorem 2. Let $\beta$ be a totally real cubic Pisot unit with Property $(\mathrm{F})$; let $\left(G_{n}\right)_{n \geq 0}$ be the sequence of best approximations of the vector $\left(1 / \beta, 1 / \beta^{2}\right)$ for the Rauzy norm and let $t(n)$ be the length of the $R$-ary digital expansion of $G_{n}$. Then $t(n) \rightarrow \infty$ as $n \rightarrow \infty$.

2.2. Rauzy fractal. Let $N \in \mathbb{Z}^{+}$and $\left(\varepsilon_{j}\right)_{k(N) \geq j \geq 0}$ be its $R$-ary expansion. We put

$$
\delta(N)=N\left(1 / \beta, 1 / \beta^{2}\right)-\left(P_{N}, Q_{N}\right)
$$


where

$$
P_{N}=\sum_{j=1}^{k(N)} \varepsilon_{j} R_{j-1}, \quad Q_{N}=\sum_{j=2}^{k(N)} \varepsilon_{j} R_{j-2}
$$

Let

$$
B=\left(\begin{array}{cc}
-b / \beta & -1 / \beta \\
1-b / \beta^{2} & -1 / \beta^{2}
\end{array}\right)
$$

LEMMA 3.

$$
B\left(\begin{array}{c}
R_{n} / \beta-R_{n-1} \\
R_{n} / \beta^{2}-R_{n-2}
\end{array}\right)=\left(\begin{array}{c}
R_{n+1} / \beta-R_{n} \\
R_{n+1} / \beta^{2}-R_{n-1}
\end{array}\right) \quad \forall n \geq 0,
$$

where $\left(R_{n}\right)_{n \geq 0}$ is the sequence defined before and $R_{-1}=R_{-2}=0$.

Proof. By induction on $n$.

Corollary 1. If $N=\sum_{j=0}^{k(N)} \varepsilon_{j} R_{j}$ then $\delta(N)=\sum_{j=0}^{k(N)} \varepsilon_{j} B^{j} \delta(1)$.

Consider the set

$$
\mathcal{E}^{\prime}=\overline{\left\{\delta(N) \mid N \in \mathbb{Z}^{+}\right\}} .
$$

It is a subset of $\mathbb{R}^{2}$ called the Rauzy fractal. It was introduced by G. Rauzy in 1982 (see [Ra82]) in the case of the polynomial $x^{3}-x^{2}-x-1$. The Rauzy fractal has been studied by many mathematicians and is related to many mathematical areas, including dynamical systems and number theory.

Another way to define the Rauzy fractal is as follows. We recall that

$$
. a_{-1} a_{-2} \ldots a_{-t}
$$

is the Rényi expansion of 1 . We denote by $E_{\beta}^{\prime}$ the set of $\left(\varepsilon_{j}\right)_{j \geq 0}$ such that for all $k \in \mathbb{Z}^{+},\left(\varepsilon_{j}\right)_{k \geq j \geq 0}$ is the $R$-ary expansion of the number $\sum_{j=0}^{k} \varepsilon_{j} R_{j}$.

We recall that in this case the set $E_{\beta}^{\prime}$ is contained in the set $E_{\beta}$ of $\beta$-expansions given by the following conditions on $\left(\varepsilon_{j}\right)_{j \geq 0}$ (see $[\mathrm{Pa} 60]$ ):

$$
\varepsilon_{j} \varepsilon_{j-1} \ldots \varepsilon_{j-t+1}<_{\mathrm{lex}} a_{-1} a_{-2} \ldots a_{-t} \quad \forall j \geq t-1
$$

and

$$
\varepsilon_{j} \ldots \varepsilon_{0} \leq_{\mathrm{lex}} a_{-1} a_{-2} \ldots a_{-j-1} \quad \forall 0 \leq j<t-1 .
$$

Applying Corollary 1 , it is easy to show that

$$
\mathcal{E}^{\prime}=\left\{\sum_{j=0}^{\infty} \varepsilon_{j} B^{j} \delta(1) \mid\left(\varepsilon_{j}\right)_{j \geq 0} \in E_{\beta}^{\prime}\right\} .
$$

Now, set $\mathcal{E}=\left\{\sum_{j=0}^{\infty} \varepsilon_{j} B^{j} \delta(1) \mid\left(\varepsilon_{j}\right)_{j \geq 0} \in E_{\beta}\right\}$. Since $E_{\beta}^{\prime} \subset E_{\beta}$ and $\ldots \varepsilon_{2} \varepsilon_{1} \varepsilon_{0} 000000 \in E_{\beta}^{\prime}$ for all $\left(\varepsilon_{j}\right)_{j \geq 0} \in E_{\beta}$ (see Proposition 2), we have

$$
B^{6} \mathcal{E} \subset \mathcal{E}^{\prime} \subset \mathcal{E}
$$


Independently, Akiyama and Praggastis studied some topological properties of the set $\mathcal{E}$. In particular they proved that 0 is an interior point of $\mathcal{E}$ (see [AS98], [Ak02], [Pr92]). Since $B^{6} \mathcal{E} \subset \mathcal{E}^{\prime}$, we deduce:

Proposition 3. Let $\beta$ be a Pisot unit of degree 3 and $\mathcal{E}^{\prime}$ its Rauzy fractal. Then 0 is an interior point of $\mathcal{E}^{\prime}$.

Proposition 3 is important for the proof of Theorem 1.

REMARK 2.1. In the present paper, the natural object is $\mathcal{E}^{\prime}$ and not $\mathcal{E}$.

2.3. Rauzy norm. Let

$$
M=\left(\begin{array}{cc}
\gamma+b / \beta & 1 / \beta \\
-\alpha-b / \beta & -1 / \beta
\end{array}\right) .
$$

One can check that the matrix $B$ (defined in 2.2) is similar to the matrix $\left(\begin{array}{ll}\alpha & 0 \\ 0 & \gamma\end{array}\right)$ and satisfies

$$
M B=\left(\begin{array}{ll}
\alpha & 0 \\
0 & \gamma
\end{array}\right) M
$$

CASE 1: $\beta$ is a non-totally real Pisot number. The Rauzy norm $\mathcal{N}$ is defined by

$$
\mathcal{N}(x)=\left|(\alpha+b / \beta) x_{1}+x_{2} / \beta\right|, \quad \forall x=\left(x_{1}, x_{2}\right) \in \mathbb{R}^{2} .
$$

It is easy to check by using (2) that $\mathcal{N}(B x)=|\alpha| \mathcal{N}(x)$ for all $x \in \mathbb{R}^{2}$.

Lemma 4. Let $\left(\varepsilon_{n}\right)_{n \geq 0}$ be a $\beta$-expansion. Then

$$
\mathcal{N}\left(\sum_{n=0}^{\infty} \varepsilon_{n} B^{n} \delta(1)\right)=\mathcal{N}(\delta(1))\left|\sum_{n=0}^{\infty} \varepsilon_{n} \alpha^{n}\right| .
$$

In particular, $\mathcal{N}\left(\delta\left(R_{n}\right)\right)=\mathcal{N}(\delta(1))\left|\alpha^{n}\right|$ for all $n \geq 0$.

Proof. Immediate, using (2) and (3).

CASE 2: $\beta$ is a totally real Pisot number. The Rauzy norm $\mathcal{N}$ is defined as the euclidean norm associated to the vector $M x$ for all $x$ in $\mathbb{R}^{2}$.

Lemma 5. Let $\left(\varepsilon_{n}\right)_{n \geq 0}$ be a $\beta$-expansion. Then

$$
\mathcal{N}\left(\sum_{n \geq 0} \varepsilon_{n} B^{n} \delta(1)\right)=\sqrt{x^{2}\left(\sum_{n=0}^{\infty} \varepsilon_{n} \alpha^{n}\right)^{2}+y^{2}\left(\sum_{n=0}^{\infty} \varepsilon_{n} \gamma^{n}\right)^{2}}
$$

where $x, y$ are the coordinates of the vector $M \delta(1)$.

Proof. The proof is a simple calculation. 
3. Proof of Theorem 1. We give the proof of Theorem 1 in the case $a \geq b \geq 0$. It is straightforward to adapt it to the other two cases.

THEOREM 3. There exists a real $c>0$ such that for all $q \in \mathbb{Z}^{+}$and all $g \in \mathbb{Z}^{2}, \mathcal{N}\left(q\left(1 / \beta, 1 / \beta^{2}\right)-g\right)<c$ implies $q\left(1 / \beta, 1 / \beta^{2}\right)-g=\delta(q)$.

As a corollary, we have:

Corollary 2. There exists a real $c>0$ such that for all $q \in \mathbb{Z}^{+}$,

$$
\text { if } \mathcal{N}_{0}\left(q\left(1 / \beta, 1 / \beta^{2}\right)\right)<c \text { then } \mathcal{N}_{0}\left(q\left(1 / \beta, 1 / \beta^{2}\right)\right)=\mathcal{N}(\delta(q)) \text {. }
$$

REMARK 3.1. Theorem 3 is also true in the case where $\beta$ is totally real. It is of independent interest and an important step in the proof of Theorem 1.

For the proof of Theorem 3, we need some propositions and lemmas. We put

$$
\mathcal{K}^{\prime}=\left\{\sum_{i=0}^{\infty} \varepsilon_{i} \alpha^{i} \mid\left(\varepsilon_{i}\right)_{i \geq 0} \in E_{\beta}^{\prime}\right\}, \quad \mathcal{K}=\left\{\sum_{i=0}^{\infty} \varepsilon_{i} \alpha^{i} \mid\left(\varepsilon_{i}\right)_{i \geq 0} \in E_{\beta}\right\} .
$$

It is clear that $\mathcal{K}^{\prime} \subset \mathcal{K}$.

Proposition 4. There exists a linear bijection $f$ from $\mathbb{R}^{2}$ to $\mathbb{C}$ such that $f(\mathcal{E})=\mathcal{K}, f\left(\mathcal{E}^{\prime}\right)=\mathcal{K}^{\prime}$ and $f\left(\mathbb{Z}^{2}\right)=\alpha^{-1} \mathbb{Z}+\alpha^{-2} \mathbb{Z}$.

Proof. There exist $h, l \in \mathbb{C}$ such that $R_{n}=h \beta^{n}+l \alpha^{n}+\bar{l} \bar{\alpha}^{n}$ for all $n \in \mathbb{Z}^{+}$. Then, for all $n \in \mathbb{N}$,

$$
\delta\left(R_{n}\right)=\left(\begin{array}{c}
R_{n} / \beta-R_{n-1} \\
R_{n} / \beta^{2}-R_{n-2}
\end{array}\right)=\left(\begin{array}{c}
c \alpha^{n}+\bar{c} \overline{\alpha^{n}} \\
d \alpha^{n}+\bar{d} \overline{\alpha^{n}}
\end{array}\right)
$$

where $c=l(1 / \beta-1 / \alpha)$ and $d=l\left(1 / \beta^{2}-1 / \alpha^{2}\right)$. One can show by solving the system given by the initial values of $R_{0}, R_{1}$ and $R_{2}$ that

$$
l=\frac{\alpha^{2}}{(\alpha-\bar{\alpha})(\alpha-\beta)} .
$$

Thus

$$
c \bar{d}-d \bar{c}=\frac{\alpha^{2} \overline{\alpha^{2}}}{\bar{\alpha}-\alpha} \neq 0
$$

Therefore if we put

$$
g(z)=\left(\begin{array}{c}
c z+\bar{c} \bar{z} \\
d z+\bar{d} \bar{z}
\end{array}\right) \quad \text { for all } z \in \mathbb{C} \text { and } f=g^{-1},
$$

we obtain $f(\mathcal{E})=\mathcal{K}$ and $f\left(\mathcal{E}^{\prime}\right)=\mathcal{K}^{\prime}$. On the other hand we can prove that $g\left(\alpha^{-1}\right)=\left(\begin{array}{c}0 \\ -1\end{array}\right)$ and $g\left(\alpha^{-2}\right)=\left(\begin{array}{c}-1 \\ b\end{array}\right)$. Thus $\alpha^{-1} \mathbb{Z}+\alpha^{-2} \mathbb{Z}=f\left(\mathbb{Z}^{2}\right)$.

REMARK 3.2. When $\beta$ is a totally real Pisot number, we have a linear bijection $f$ from $\mathbb{R}^{2}$ to $\mathbb{R}^{2}$ such that $f(\mathcal{E})=\mathcal{K}, f\left(\mathcal{E}^{\prime}\right)=\mathcal{K}^{\prime}$ and 
$f\left(\mathbb{Z}^{2}\right)=\theta^{-1} \mathbb{Z}+\theta^{-2} \mathbb{Z}$, where $\mathcal{K}^{\prime}=\left\{\sum_{i=0}^{\infty} \varepsilon_{i} \theta^{i} \mid\left(\varepsilon_{i}\right)_{i \geq 0} \in E_{\beta}^{\prime}\right\}, \quad \mathcal{K}=$ $\left\{\sum_{i=0}^{\infty} \varepsilon_{i} \theta^{i} \mid\left(\varepsilon_{i}\right)_{i \geq 0} \in E_{\beta}\right\}$, and $\theta^{i}=\left(\alpha^{i}, \gamma^{i}\right)$ for all $i \geq-2$.

By Proposition 4 and the fact that 0 is an interior point of $\mathcal{E}^{\prime}$ (see 2.2), we deduce the following result.

Proposition 5. The point 0 is an interior point of $\mathcal{K}^{\prime}$.

The next step is:

Proposition 6. $\mathcal{K}^{\prime} \cap\left(\alpha^{-1} \mathbb{Z}+\alpha^{-2} \mathbb{Z}\right)=\{0\}$.

The proof of Proposition 6 is postponed to Section 5.

Remark 3.3. For $\mathcal{K}$, the last statement is not true since $-\alpha^{-1} \in \mathcal{K}$.

Proof of Theorem 3. By Propositions 4 and 6, we deduce that $\mathcal{E}^{\prime} \cap \mathbb{Z}^{2}$ $=\{0\}$. Therefore there exists a real number $c>0$ such that for all $g \in \mathbb{Z}^{2}, \inf _{x \in \mathcal{E}^{\prime}} \mathcal{N}(g-x)<c \Rightarrow g=0$. Assume that $\mathcal{N}\left(q\left(1 / \beta, 1 / \beta^{2}\right)-g\right)<c$. Since $\delta(q)-q\left(1 / \beta, 1 / \beta^{2}\right) \in \mathbb{Z}^{2}$ and $\delta(q) \in \mathcal{E}^{\prime}$, it follows that $\mathcal{N}(\delta(q))-(\delta(q)-$ $\left.q\left(1 / \beta, 1 / \beta^{2}\right)-g\right)<c$. Hence $\delta(q)-q\left(1 / \beta, 1 / \beta^{2}\right)+g=0$, which completes the proof of Theorem 3 .

Proposition 7. Let $n \in \mathbb{N}$ and $0<q<R_{n}$. Then $\mathcal{N}\left(\delta\left(R_{n}\right)\right)<\mathcal{N}(\delta(q))$.

Proof. It is sufficient to prove the assertion for $R_{n-1} \leq q<R_{n}$. Assume that $q=\sum_{k=0}^{n-1} \varepsilon_{k} R_{k}$ where $\left(\varepsilon_{k}\right)_{n-1 \geq k \geq 0} \in E_{\beta}^{\prime}$ and $\varepsilon_{n-1} \neq 0$. By Proposition $4, \mathcal{N}\left(\delta\left(R_{n}\right)\right)<\mathcal{N}(\delta(q))$ is equivalent to $\left|\alpha^{n}\right|<\left|\sum_{k=0}^{n-1} \varepsilon_{k} \alpha^{k}\right|$.

On the other hand, let $x_{n-1}=\sum_{k=0}^{n-1} \varepsilon_{k} \alpha^{k}$. The number $x_{n-1}$ is an algebraic integer. Then if we put $z_{k}=\sum_{k=0}^{n-1} \varepsilon_{k} \beta^{k}$, we have $\left|x_{k}\right|^{2} z_{k} \in \mathbb{Z}$. Since $z_{k}$ and $x_{k}$ are not 0 , we have $\left|x_{k}\right|^{2} \geq 1 / z_{k}$. Since $\left(\varepsilon_{k}\right)_{n-1 \geq k \geq 0}$ is a $\beta$-expansion, by Lemma 1 we have $0<z_{k}<\beta^{n}$. Therefore

$$
\left|x_{k}\right|^{2}>1 / \beta^{n}=|\alpha|^{2 n} \text {. }
$$

Hence $\left|\alpha^{n}\right|<\left|\sum_{k=0}^{n-1} \varepsilon_{k} \alpha^{k}\right|$.

End of proof of Theorem 1 in the case $a \geq b \geq 0$. Now, we will prove that there exists $n_{0} \in \mathbb{N}$ such that $\left(R_{n}\right)_{n \geq n_{0}}$ is the best approximation of the vector $\left(1 / \beta, 1 / \beta^{2}\right)$ for the norm $\mathcal{N}$.

Let $c$ be the real number defined in Theorem 3. Let $n_{0} \in \mathbb{N}$ be such that $\mathcal{N}\left(\delta\left(R_{n_{0}}\right)\right)=\left|\alpha^{n_{0}}\right|<c$. Let $n \geq n_{0}$ and $0<q<R_{n}$. Assume

$$
\mathcal{N}_{0}\left(q\left(1 / \beta, 1 / \beta^{2}\right)\right) \leq \mathcal{N}_{0}\left(R_{n}\left(1 / \beta, 1 / \beta^{2}\right)\right)<c .
$$

By Corollary $2, \mathcal{N}_{0}\left(q\left(1 / \beta, 1 / \beta^{2}\right)\right)=\mathcal{N}(\delta(q))$ and $\mathcal{N}_{0}\left(R_{n}\left(1 / \beta, 1 / \beta^{2}\right)\right)=$ $\mathcal{N}\left(\delta\left(R_{n}\right)\right)$. Thus $\mathcal{N}(\delta(q)) \leq \mathcal{N}\left(\delta\left(R_{n}\right)\right)$. This contradicts Proposition 7 .

REMARK 3.4. The proofs in the cases $b=a+1$ and $b=-1, a \geq 2$ are mutatis mutandis analogous to the previous one. 
4. Proof of Theorem 2. We give a proof of Theorem 2 in the case $a \geq b \geq 0$. It is straightforward to adapt it to the other two cases.

Let $\beta$ be a Pisot unit of degree 3 , and let $\alpha, \gamma$ be its real Galois conjugates. Let $\left(G_{n}\right)_{n \geq 0}$ be the sequence of best approximations of the vector $\left(1 / \beta, 1 / \beta^{2}\right)$ for the norm $\mathcal{N}$. Assume that $\alpha>0$. Since $1=a_{-1} / \beta+\cdots+a_{-t} / \beta^{t}$ where $a_{-1} \ldots a_{-t}=d(1, \beta)$, and $\alpha$ is a Galois conjugate of $\gamma$, we have $1=a_{-1} / \alpha+\cdots+a_{-t} / \alpha^{t}$, which is impossible because $\alpha<\beta$. Hence $\alpha$ and $\gamma$ belong to $]-1,0[$. Without loss of generality, we assume that $-1<\alpha<$ $\gamma<0$. This means that $0<|\gamma|<|\alpha|<1$.

By contradiction, if the sequence $(t(n))_{n \geq 0}$ does not tend to infinity, there exists an integer $M \in \mathbb{N}$ and a subsequence $\left(t\left(n_{u}\right)\right)_{u \geq 0}$ such that $t\left(n_{u}\right) \leq M$ for all $u$. So, for all $u$, there exist $k_{u}$ and a $\beta$-expansion $\varepsilon_{k_{u}+M-1} \ldots \varepsilon_{k_{u}}$ such that $\varepsilon_{k_{u}} \geq 1$ and

$$
G_{n_{u}}=\sum_{i=k_{u}}^{k_{u}+M-1} \varepsilon_{i} R_{i}
$$

By Theorem 3, we deduce that for $n$ large enough,

$$
\mathcal{N}_{0}\left(G_{n}\left(1 / \beta, 1 / \beta^{2}\right)\right)=\mathcal{N}\left(\delta\left(G_{n}\right)\right) .
$$

In what follows, we will assume that $u$ is so large that $G_{n_{u}}$ satisfies (6).

For all $d \in \mathbb{N}$,

$$
\mathcal{N}\left(\delta\left(R_{d}\right)\right)=\sqrt{x^{2} \alpha^{2 d}+y^{2} \gamma^{2 d}},
$$

where $x, y$ are the coordinates of the vector $\delta(1)$. As all norms are equivalent on $\mathbb{R}^{2}$, there exists a constant $C>1$ such that, for all $v=\left(v_{1}, v_{2}\right) \in \mathbb{R}^{2}$,

$$
\frac{1}{C} \max \left(\left|v_{1}\right|,\left|v_{2}\right|\right) \leq \mathcal{N}(v) \leq C \max \left(\left|v_{1}\right|,\left|v_{2}\right|\right) .
$$

So there is $T>0$ such that $\mathcal{N}\left(\delta\left(R_{d}\right)\right) \leq T\left|\alpha^{d}\right|$ for all $d \in \mathbb{N}$. Moreover, for all $u, G_{n_{u}} \geq R_{k_{u}}$. As $G_{n_{u}}$ belongs to the sequence of best approximations, we have

$$
\mathcal{N}\left(\delta\left(G_{n_{u}}\right)\right) \leq \mathcal{N}\left(\delta\left(R_{k_{u}}\right)\right) \leq T\left|\alpha^{k_{u}}\right| .
$$

On the other hand, by (7),

$$
\mathcal{N}\left(\delta\left(G_{n_{u}}\right)\right) \geq \frac{1}{C} \max \left(\left|\sum_{i=k_{u}}^{k_{u}+M-1} \varepsilon_{i} \alpha^{i}\right|,\left|\sum_{i=k_{u}}^{k_{u}+M-1} \varepsilon_{i} \gamma^{i}\right|\right) \geq \frac{1}{C}\left|\sum_{i=k_{u}}^{k_{u}+M-1} \varepsilon_{i} \alpha^{i}\right| .
$$

Set $K(M)=\min \left(\left|\sum_{i=0}^{M-1} \varepsilon_{i} \alpha^{i}\right|\right)$ where the minimum is taken over all $\beta$-expansions $\varepsilon_{M-1} \ldots \varepsilon_{0}$ of length $M$ with $\varepsilon_{0} \geq 1$. Then

$$
\mathcal{N}\left(\delta\left(G_{n_{u}}\right)\right) \geq \frac{K(M)}{C}\left|\alpha^{k_{u}}\right| .
$$


We have proven that

$$
\frac{K(M)}{C}\left|\alpha^{k_{u}}\right| \leq \mathcal{N}\left(\delta\left(G_{n_{u}}\right)\right) \leq T\left|\alpha^{k_{u}}\right|
$$

Now we give an estimate of $k_{u}$ in terms of $G_{n_{u}}$. As $\beta$ is a Pisot number, there exists a constant $\kappa$ such that for all $u$,

$$
\frac{1}{\kappa} \beta^{k_{u}} \leq R_{k_{u}} \leq G_{n_{u}} \leq R_{k_{u}+M} \leq \kappa \beta^{k_{u}+M}
$$

So there are two positive constants $C_{1}, C_{2}$ such that

$$
\frac{\log \left(G_{n_{u}}\right)}{\log (\beta)}-C_{1} \leq k_{u} \leq \frac{\log \left(G_{n_{u}}\right)}{\log (\beta)}+C_{2} .
$$

Combining (8) and (9), we obtain

$$
\begin{gathered}
\frac{K(M)}{C}|\alpha|^{C_{2}}\left(\frac{1}{G_{n_{u}}}\right)^{\log (1 /|\alpha|) / \log (\beta)} \\
\leq \mathcal{N}\left(\delta\left(G_{n_{u}}\right)\right) \leq T|1 / \alpha|^{C_{1}}\left(\frac{1}{G_{n_{u}}}\right)^{\log (1 /|\alpha|) / \log (\beta)} .
\end{gathered}
$$

As $(1 /|\alpha|)(1 /|\gamma|)=\beta$ and $1 /|\alpha|<1 /|\gamma|$, we have

$$
\frac{\log (1 /|\alpha|)}{\log (\beta)}<1 / 2 \text {. }
$$

But $\left(G_{n}\right)_{n \geq 0}$ is the sequence of best approximations of $\left(1 / \beta, 1 / \beta^{2}\right)$. So, by the Dirichlet theorem (see, for instance, [Ca57, p. 13]), there exists a constant $C_{3}$ such that, for all $n \in \mathbb{N}$,

$$
\mathcal{N}_{0}\left(G_{n}\left(1 / \beta, 1 / \beta^{2}\right)\right) \leq \frac{C_{3}}{G_{n}^{1 / 2}} .
$$

Consequently, for $u$ large enough,

$$
\mathcal{N}_{0}\left(G_{n_{u}}\left(1 / \beta, 1 / \beta^{2}\right)\right) \leq \frac{C_{3}}{G_{n_{u}}^{1 / 2}} .
$$

Inequality (12) contradicts (10) and (11). So Theorem 2 is proven.

REMARK 4.1. Theorem 2 is also true if $\beta$ is a Pisot unit of degree $d \geq 4$ which has Property (F).

5. Proof of Proposition 6. The proof of Proposition 6 requires several propositions and lemmas.

Proposition 8. Let $x=\sum_{i=0}^{\infty} \varepsilon_{i} \alpha^{i}$ and $y=\sum_{i=0}^{\infty} \varepsilon_{i}^{\prime} \alpha^{i}$ where $\left(\varepsilon_{i}\right)_{i \geq 0}$, $\left(\varepsilon_{i}^{\prime}\right)_{i \geq 0} \in E_{\beta}$. Then $x=y$ if and only if the set $\left\{\alpha^{-k} \sum_{i=0}^{k}\left(\varepsilon_{i}-\varepsilon_{i}^{\prime}\right) \alpha^{i} \mid k \geq 0\right\}$ is finite. 
A proof of this proposition in the case of Tribonacci numbers can be found in [Me00]. Here, we apply the tools developed by Thurston [Th90].

Proof of Proposition 8. The "if" part is obvious because if there exists a fixed number $M$ such that $\left|\alpha^{-k} \sum_{i=0}^{k}\left(\varepsilon_{i}-\varepsilon_{i}^{\prime}\right) \alpha^{i}\right| \leq M$ for all $k \geq 0$, then $x=y$ since $|\alpha|<1$. Now assume that $x=y$ and for an integer $k$, define $A_{k}=\sum_{i=0}^{k}\left(\varepsilon_{i}-\varepsilon_{i}^{\prime}\right) \alpha^{i-k}$. Since $\beta$ has Property $(\mathrm{F})$, there exists a finite $\beta$-expansion $\left(c_{i}\right)_{M \geq i \geq L}$ such that $A_{k}= \pm \sum_{i=L}^{M} c_{i} \alpha^{i}$. Assume without loss of generality that $A_{k}=\sum_{i=L}^{M} c_{i} \alpha^{i}$. Then $\sum_{i=0}^{k} \varepsilon_{i} \beta^{i-k}=\sum_{i=0}^{k} \varepsilon_{i}^{\prime} \beta^{i-k}+$ $\sum_{i=L}^{M} c_{i} \beta^{i}$. Therefore $M \leq 0$, since otherwise, by Lemma $1, \sum_{i=0}^{k} \varepsilon_{i} \beta^{i-k}<$ $\sum_{i=L}^{M} c_{i} \beta^{i}$. On the other hand, since $x=y$,

$$
A_{k}=\sum_{i=k+1}^{\infty}\left(\varepsilon_{i}^{\prime}-\varepsilon_{i}\right) \alpha^{i-k}=\sum_{i=1}^{\infty} \varepsilon_{k+i}^{\prime} \alpha^{i}-\sum_{i=1}^{\infty} \varepsilon_{k+i} \alpha^{i} .
$$

The hypothesis implies that there exists a fixed constant $d(\beta)=d>0$ such that

$$
\left|A_{k}\right|<d, \quad \forall k \geq 0 .
$$

Now put $z_{k}=\beta^{-k} \sum_{i=0}^{k}\left(\varepsilon_{i}-\varepsilon_{i}^{\prime}\right) \beta^{i}$ for all $k \geq 0$. Since $1 / \beta$ is an algebraic integer, for all $k \geq 0, z_{k}$ is also an algebraic integer in $\mathbb{Q}[\beta]$. The Galois conjugates of $z_{k}$ are contained in the set $\left\{\beta_{j}^{-k} \sum_{i=0}^{k}\left(\varepsilon_{i}-\varepsilon_{i}^{\prime}\right) \beta_{j}^{i} \mid j=1,2,3\right\}$, where $\beta_{1}=\beta, \beta_{2}=\alpha, \beta_{3}=\bar{\alpha}$.

By (13) and the fact that $z_{k}$ is bounded by a constant independent of $k$, we deduce that the set $\left\{A_{k} \mid k \geq 0\right\}$ is finite.

Lemma 6 ([FS92]). Let $\beta$ be a Pisot real number. There exists $S=S(\beta)$ with the following property. Let $x, y \in \operatorname{Fin}_{N}(\beta), x>y$. If $x+y \in \operatorname{Fin}(\beta)$ then $x+y \in \operatorname{Fin}_{N+S}(\beta)$, and if $x-y \in \operatorname{Fin}(\beta)$ then $x-y \in \operatorname{Fin}_{N+S}(\beta)$.

Lemma 7. Let $\left(c_{i}\right)_{i \geq 0}$ be an element of $E_{\beta}$ and $\left(d_{i}\right)_{P \geq i \geq 0}$ be a finite $\beta$-expansion. If $\sum_{i=0}^{\infty} c_{i} \alpha^{i}=\sum_{i=0}^{P} d_{i} \alpha^{i}$ then $c_{i}=d_{i}$ for all $0 \leq i \leq P$, and $c_{i}=0$ for all $i>P$.

Proof. Put $X=\left\{i \in \mathbb{N} \mid c_{i} \neq 0\right\}$. Assume that $X$ is a finite set; then there exists $N \in \mathbb{N}$ such that $c_{i}=0$ for all $i>N$. Since $\beta$ is a Galois conjugate of $\alpha$, we deduce that $\sum_{i=0}^{N} c_{i} \beta^{i}=\sum_{i=0}^{P} d_{i} \beta^{i}$. Hence, by Lemma 1 , we obtain $N=P$ and $c_{i}=d_{i}$ for all $0 \leq i \leq P$.

Assume that $X$ is an infinite set and put $d_{i}=0$ for all $i>P$. For all $k \in X$, put $A_{k}=\sum_{i=0}^{k} c_{i} \alpha^{i}$ and $B=\sum_{i=0}^{P} d_{i} \alpha^{i}$. By Proposition 8, the set $V=\left\{\alpha^{-k} \sum_{i=0}^{k}\left(c_{i}-d_{i}\right) \alpha^{i} \mid k \geq 0\right\}$ is finite, so there exists an integer $L$, as large as we want, such that for some $k \geq P, \alpha^{-k}\left(A_{k}-B\right)=$ 
$\alpha^{-k-L}\left(A_{k+L}-B\right)$. Then

$$
\sum_{i=0}^{k+L} c_{i} \alpha^{i}=\left(\sum_{i=L}^{k+L} c_{i-L} \alpha^{i}-\sum_{i=L}^{L+P} d_{i-L} \alpha^{i}\right)+\sum_{i=0}^{P} d_{i} \alpha^{i}
$$

By Lemma 6 and the fact that $\sum_{i=L}^{k+L} c_{i-L} \beta^{i}-\sum_{i=L}^{L+P} d_{i-l} \beta^{i}>0$, there exists an integer $S=S(\beta)>0$ such that

$$
\sum_{i=L}^{k+L} c_{i-L} \alpha^{i}-\sum_{i=L}^{L+P} d_{i-L} \alpha^{i}=\sum_{i=L-S}^{R} e_{i} \alpha^{i},
$$

where $\left(e_{i}\right)_{R \geq i \geq L-S} \in E_{\beta}$. We deduce that

$$
\sum_{i=0}^{P} d_{i} \alpha^{i}+\sum_{i=L-S}^{R} e_{i} \alpha^{i}=\sum_{i=0}^{k+L} c_{i} \alpha^{i}
$$

If we choose $L$ such that $L-S-P$ is large enough to guarantee that the word $e_{R} \ldots e_{L-S} 0 \ldots 0 d_{P} \ldots d_{0}$ is a $\beta$-expansion, we infer by Lemma 1 that $c_{i}=d_{i}$ for all $0 \leq i \leq P$. Hence $\sum_{i=P+1}^{\infty} c_{i} \alpha^{i}=0$ and then by applying the same argument we conclude that $c_{i}=0$ for all $i>P$.

REMARK 5.1. The previous lemma also follows from Theorem 2 in [Ak99].

Proof of Proposition 6. Let $z=p_{1} \alpha^{-1}+p_{2} \alpha^{-2}, p_{1}, p_{2} \in \mathbb{Z}$. Assume that

$$
z=\sum_{i=0}^{\infty} \varepsilon_{i} \alpha^{i}
$$

where $\left(\varepsilon_{i}\right)_{i \geq 0} \in E_{\beta}^{\prime}$. We divide the proof into two cases.

CAsE 1: $p_{1} \beta^{-1}+p_{2} \beta^{-2}>0$. Since $\beta$ has Property $(\mathrm{F})$, there exists a $\beta$-expansion $\left(a_{i}\right)_{N \geq i \geq M}$ with $a_{M} \neq 0$ such that

$$
p_{1} \beta^{-1}+p_{2} \beta^{-2}=\sum_{i=M}^{N} a_{i} \beta^{i} \text {. }
$$

Therefore

$$
\sum_{i=0}^{\infty} \varepsilon_{i} \alpha^{i}=\sum_{i=M}^{N} a_{i} \alpha^{i}
$$

Multiplying both sides of (17) by $\alpha^{-M}$ and using Lemma 7 , we deduce that $M \geq 0$ and $a_{i}=\varepsilon_{i}$ for all $M \leq i \leq N$. By (16) and Lemma 2, we have

$$
p_{1} \beta^{-1}+p_{2} \beta^{-2}=\sum_{i=M}^{N} a_{i} R_{i}+\sum_{i=M}^{N} a_{i}\left(b R_{i-1}+R_{i-2}\right) / \beta+\sum_{i=M}^{N} a_{i} R_{i-1} / \beta^{2} .
$$

Therefore $a_{M}=0$. This leads to a contradiction. 
$\mathrm{CASE} 2: p_{1} \beta^{-1}+p_{2} \beta^{-2}<0$. By $(15), \sum_{i=0}^{k} \varepsilon_{i} \alpha^{i}-p_{1} \alpha^{-1}-p_{2} \alpha^{-2}$ converges to 0 as $k \rightarrow \infty$. Since $0 \in \operatorname{int}\left(\mathcal{K}^{\prime}\right)$, for $k$ large enough

$$
\sum_{i=0}^{k} \varepsilon_{i} \alpha^{i}-p_{1} \alpha^{-1}-p_{2} \alpha^{-2} \in \mathcal{K}^{\prime} .
$$

We fix $k$ satisfying $(*)$; then there exist $\left(d_{i}\right)_{i \geq 0} \in E_{\beta}^{\prime}$ such that

$$
\sum_{i=0}^{k} \varepsilon_{i} \alpha^{i}-p_{1} \alpha^{-1}-p_{2} \alpha^{-2}=\sum_{i=0}^{\infty} d_{i} \alpha^{i} .
$$

On the other hand, since $\sum_{i=0}^{k} \varepsilon_{i} \beta^{i}-p_{1} \beta^{-1}-p_{2} \beta^{-2}>0$ and $\beta$ has Property $(\mathrm{F})$, there exists a $\beta$-expansion $\left(b_{i}\right)_{M \geq i \geq L}$ such that $\sum_{i=L}^{M} b_{i} \alpha^{i}=$ $\sum_{i=0}^{\infty} d_{i} \alpha^{i}$. This implies by Lemma 7 that $d_{i}=0$ for all $i>M$. Thus

$$
\sum_{i=0}^{k} \varepsilon_{i} \alpha^{i}-p_{1} \alpha^{-1}-p_{2} \alpha^{-2}=\sum_{i=0}^{M} d_{i} \alpha^{i} .
$$

Using Lemma 2, we deduce that

$$
\begin{aligned}
\sum_{i=0}^{k} \varepsilon_{i} R_{i}+\left(\sum_{i=0}^{k} \varepsilon_{i}\left(b R_{i-1}+R_{i-2}\right)-p_{1}\right) / \alpha+\sum_{i=0}^{k}\left(\varepsilon_{i} R_{i-1}-p_{2}\right) / \alpha^{2} \\
=\sum_{i=0}^{M} d_{i} R_{i}+\sum_{i=0}^{M} d_{i}\left(b R_{i-1}+R_{i-2}\right) / \alpha+\sum_{i=0}^{k} d_{i} R_{i-1} / \alpha^{2} .
\end{aligned}
$$

Since $\alpha$ is an algebraic integer of degree 3 , we find that $\sum_{i=0}^{k} \varepsilon_{i} R_{i}=$ $\sum_{i=0}^{M} d_{i} R_{i}$. We deduce that $\varepsilon_{i}=d_{i}$ for all $i$. Hence $p_{1}=p_{2}=0$, which leads to a contradiction.

\section{References}

[Ak99] S. Akiyama, Self affine tiling and Pisot numeration system, in: Number Theory and Its Applications (Kyoto, 1997), Dev. Math. 2, Kluwer, Dordrecht, 1999, $7-17$.

[Ak00] -, Cubic Pisot units with finite beta expansions, in: Algebraic Number Theory and Diophantine Analysis (Graz, 1998), de Gruyter, 2000, 11-26.

[Ak02] - On the boundary of self affine tiling generated by Pisot numbers, J. Math. Soc. Japan 54 (2002), 283-308.

[AS98] S. Akiyama and T. Sadahiro, A self-similar tiling generated by the minimal Pisot number, Acta Math. Info. Univ. Ostraviensis 6 (1998), 9-26.

[Ca57] J. W. S. Cassels, An Introduction to Diophantine Approximation, Cambridge Tracts in Math. and Math. Phys. 45, Cambridge Univ. Press, New York, 1957.

[Ch99] N. Chevallier, Best simultaneous Diophantine approximations of some cubic algebraic numbers, J. Théor. Nombres Bordeaux 14 (2002), 403-414. 
[CHM01] N. Chekhova, P. Hubert et A. Messaoudi, Propriétés combinatoires, ergodiques et arithmétiques de la substitution de Tribonacci, ibid. 13 (2001), 371-394.

[F92] C. Frougny, Representation of numbers and finite automata, Math. Systems Theory 25 (1992), 37-60.

[FS92] C. Frougny and B. Solomyak, Finite Beta-expansions, Ergodic Theory Dynam. Systems 12 (1992), 713-723.

[La82] J. C. Lagarias, Best simultaneous Diophantine approximations II. Behavior of consecutive best approximations, Pacific J. Math. 102 (1982), 61-88.

[Me00] A. Messaoudi, Frontière du fractal de Rauzy et système de numération complexe, Acta Arith. 95 (2000), 195-224.

[Pa60] W. Parry, On the $\beta$-expansions of real numbers, Acta Math. Acad. Sci. Hungar. 11 (1960), 401-416.

[Pr92] B. Praggastis, Markov partitions for hyperbolic toral automorphisms, Ph.D. thesis, Univ. of Washington, 1992.

[Ra82] G. Rauzy, Nombres algébriques et substitutions, Bull. Soc. Math. France 110 (1982), 147-178.

[Re57] A. Rényi, Representations of real numbers and their ergodic properties, Acta Math. Acad. Sci. Hungar. 8 (1957), 477-493.

[Th90] W. P. Thurston, Groups, Tilings, and Finite State Automata, AMS Colloquium lectures, 1990.

Laboratoire d'Analyse, Topologie

et Probabilités

Faculté des Sciences de Saint Jérôme

Avenue Escadrille Normandie-Niemen

13397 Marseille Cedex 20, France

E-mail: hubert@cmi.univ-mrs.fr
Departamento de matemática UNESP-Universidade Estadual Paulista Rua Cristovão Colombo 2265, Jardim Nazareth

CEP 15054-000

São José de Rio Preto, SP, Brasil E-mail: messaoud@ibilce.unesp.br

Received on 29.6.2004

and in revised form on 6.3.2006 